\title{
Two-photon Bio-imaging with a Mode-locked Semiconductor Laser
}

\author{
Hengchang Guo, Ki-ichi Sato, Keijiro Takashima, and Hiroyuki Yokoyama \\ New Industry Creation Hatchery Center (NICHe), Tohoku University, Sendai 980-8579, Japan; \\ hcguo@niche.tohoku.ac.jp, yoko@niche.tohoku.ac.jp
}

\begin{abstract}
We demonstrated two-photon imaging of biological tissues by employing a mode-locked semiconductor laser. Kilowatt-peak-power second-harmonic pulses were obtained from amplified 1.55- $\mu \mathrm{m}$ optical pulses, and were used for two-photon excitation. (C)2006 Optical Society of America
\end{abstract}

OCIS codes: (140.5960) Semiconductor lasers; (170.3880) Medical and biological imaging.

\section{Introduction}

Picosecond and femtosecond optical pulses have been widely used in biophotonics, such as multi-photon imaging and coherent anti-Stokes Raman scattering (CARS) [1,2]. As excitation optical pulse source, mode-locked Ti:sapphire laser is generally used as a high-peak-power optical pulse source [1,2]. However, in view of reliable and cost-effective instruments, compact and turn-key light sources are strongly desirable.

In this paper, we report a simple-scheme kilowatt-peak-power picosecond optical pulse source at a wavelength of $0.77-\mu \mathrm{m}$ using a mode-locked laser diode (MLLD). And these high-peak-power optical pulses were successfully used for two-photon bio-imaging of mouse kidney tissues.

\section{Kilowatt-peak-power optical pulse generation}

The experimental configuration for kilowatt-peak-power optical pulse generation and two-photon microscopy is shown in Fig. 1. Picosecond optical pulses are generated by an external cavity MLLD at an operation wavelength of $1548-\mathrm{nm}$, repetition rate of $500-\mathrm{MHz}$ and pulse width of $2.5 \mathrm{ps}$. We developed an electric pulse generator for pre-scale gating operation of a semiconductor optical amplifier (SOA), and extracted the optical pulses mainly at 1-MHz. In addition, the SOA also worked as a pre-amplifier of optical pulses.

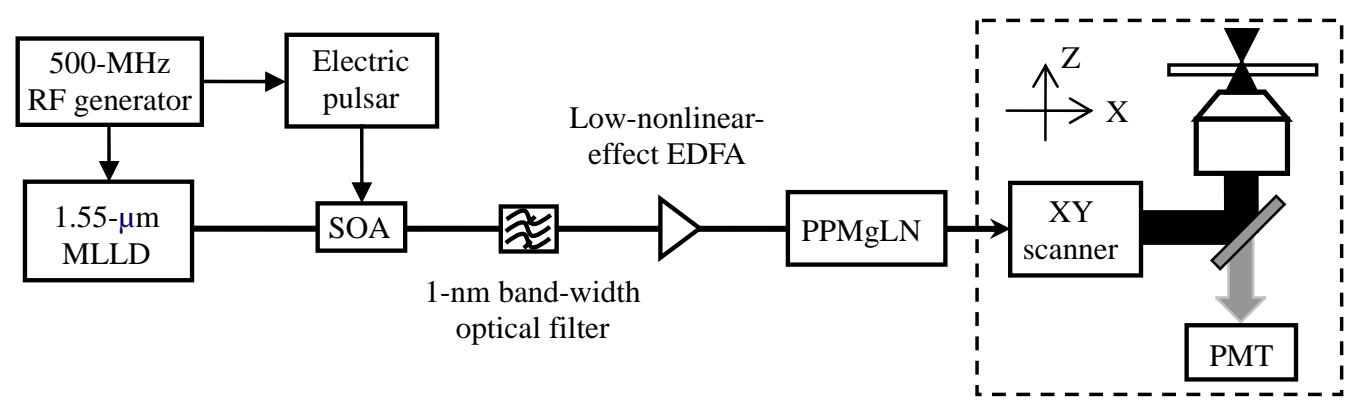

Fig.1. Schematic of kilowatt-peak-power picosecond optical pulse generation and two-photon microscopy. Two-photon fluorescence microscope section is indicated in the dashed-line-surrounding area.

After the SOA, a 1-nm band-width optical filter was inserted. This filter broadened the optical pulse width to 3.5-ps, and simultaneously removed spontaneous emission noise. As a main amplifier, we employed a low-nonlinear-effect erbium-doped fiber amplifier (EDFA) specially designed to avoid serious spectral distortions by self-phase-modulation (SPM) inside EDFA [3]. By this optical amplifier, the maximum average 
output power of approximately 13-mW was obtained. With the pulse duration of 3.5-ps and pulse repetition rate of 1-MHz, the optical peak-power reached $3.7 \mathrm{~kW}$.

From the output of EDFA, 1548-nm optical-pulses were converted to second-harmonic (SH) light by a periodically-poled MgO-doped $\mathrm{LiNbO}_{3}(\mathrm{PPMgLN})$ device [4], and then 3.6-mW average-power SH optical pulses was obtained. The SHG power conversion efficiency was over $25 \%$. Since the temporal width of SH optical pulses were measured to be 3-ps, the 774-nm optical pulse peak-power reached $1.2 \mathrm{~kW}$. This peak-power was high enough for two-photon bio-imaging.

It should be emphasized that, in the present configuration, there were not any high average-power optical devices. The mechanism we can obtain high peak-power optical-pulses is attributed to a long upper-laser-level lifetime of several milliseconds for EDFA. If the repetition rate of the incident optical pulses is decreased, the energy stored in the EDFA during the pulse interval time is increased, and each optical pulse can receive higher saturation energy after amplification.

\section{Two-photon bio-imaging}

We directed the 774-nm optical pulses into a fluorescence microscope (Olympus IX71) modified for two-photon imaging. The focus of laser beam is scanned on XY plane by a two perpendicular-axis pair of galvanometer mirrors into the inverted-microscope. In order to change the focal point depth in the specimen, a stepping motor was used. Finally, the beam is focused by a $60 \times(\mathrm{NA}=1.2)$ water-immersion objective lens onto a specimen.

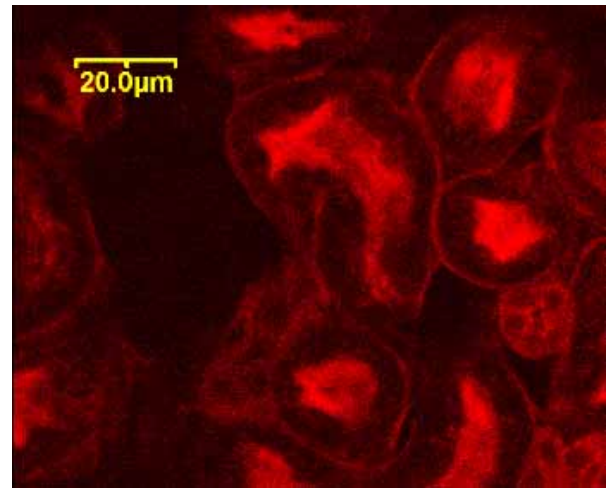

Fig.1. Two-photon excited fluorescence intensity image of convoluted tubules in mouse kidney stained with Alexa Fluor 488 wheat germ agglutinin.

For bio-imaging specimen, we prepared a section of mouse kidney stained with Alexa Fluor 488 wheat germ agglutinin, a green-fluorescent lectin. The fluorescence dye was used to label elements of the glomeruli and convoluted tubules. As illustrated in Fig. 2, we obtained a clear image of two-photon fluorescence, and the present result indicates the usefulness of our new-scheme high-peak-power picosecond-optical-pulse-source for nonlinear bio-imaging.

\section{Conclusions}

In conclusion, we have carried out the generation of multi-kilowatt 1.55- $\mu$ m picosecond optical pulses using a combination of a mode-locked semiconductor laser, a gating SOA, and a low-average-power fiber-amplifier. Using a PPMgLN crystal, second-harmonic optical pulses of 774-nm wavelength and 3-ps duration were generated with the maximal conversion efficiency of over 25\%; the SH optical-pulse peak-power exceeded $1.2-\mathrm{kW}$ at a repetition rate of 1-MHz. Subsequently, employing these SH optical pulses, we successfully demonstrated high-resolution and high-contrast two-photon bio-imaging for mouse kidney tissues stained with Alexa Fluo 488 wheat germ agglutinin. Our present result will stimulate many biophotonic applications of stable and compact semiconductor laser. 


\section{References}

1. P.F. Curley, A.I. Ferguson, J.G. White, W.B. Amos, “Application of a femtosecond self-sustained mode-locked Ti:sapphire laser to the field of laser scanning confocal microscopy”, Opt. Quantum Electron. 24, 851-859 (1992).

2. A. Zumbush, G.R. Holtom, X.S. Xie, “Three-Dimensional Vibrational Imaging by Coherent Anti-Stokes Raman Scattering”, Phy. Rev. Lett. 82, 4142-4145 (1999)

3. H. Yokoyama, M. Shirane, Y. Sasaki, H. Ito, H. Taniguchi, "Supercontinuum generation in 800-nm wavelength region with semiconductor laser pulses”, presented at the Nonlinear Optics, ThB3, Waikoloa, Hawaii, Aug. 2004.

4. T. Yoda, H. Yokoyama, K. Sato, H. Taniguchi, H. Ito, "High-peak-power picosecond optical-pulse generation with a gain-switched semiconductor laser, and high-efficiency wavelength convertion”, presented at the Pacific Rim Conference on Lasers and Electro-Optics (CLEO-PR), CFM2-5, Tokyo, Japan, Jul. 2005. 